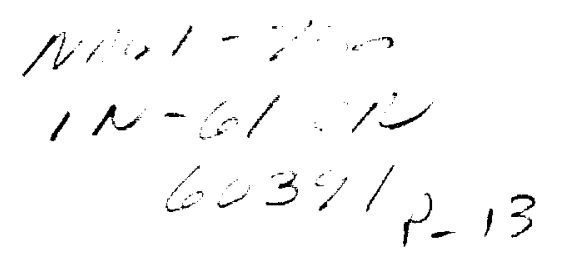

CU-CSSC-91-25

CENTER FOR SPACE STRUCTURES AND CONTROLS

\title{
THE INDIVIDUAL ELEMENT TEST REVISITED
}

(NASA-CR-199039) THE INOIVICUAL ELEMENT TEST REVISITED (COIOR AdO N95-32215 Univ.) $13 \mathrm{p}$

Unclas

G3/61 0060391

\begin{tabular}{|c|c|}
\hline & $\begin{array}{l}\text { by } \\
\text { C. Militello and C. A. Felippa }\end{array}$ \\
\hline August 1991 & $\begin{array}{l}\text { COLLEGE OF ENGINEERING } \\
\text { UNIVERSITY OF COLORADO } \\
\text { CAMPUS BOX } 429 \\
\text { BOULDER, COLORADO } 80309\end{array}$ \\
\hline
\end{tabular}




\title{
The Individual Element Test Revisited
}

\author{
Carmelo Militello and Carlos A. Felippa \\ Department of Aerospace Engineering Sciences and \\ Center for Space Structures and Controls \\ University of Colorado \\ Boulder, Colorado 80309-0429, USA
}

August 1991

Report No. CU-CSSC-91-25

Invited contribution to The Finite Element Method in the 90's, a volume to be published in honor of the 70th anniversary of Professor O. C. Zienkiewicz.

Research supported by NASA Langley Research Center under Grant NAG1-756. 


\title{
THE INDIVIDUAL ELEMENT TEST REVISITED
}

\author{
Carmelo Militello and Carlos A. Felippa \\ Department of Aerospace Engineering Sciences \\ and Center for Space Structures and Controls \\ University of Colorado at Boulder \\ Boulder, Colorado 80309-0429, USA
}

\section{SUMMARY}

The subject of the patch test for finite elements retains several unsettled aspects. In particular, the issue of one-element versus multielement tests needs clarification. Following a brief historical review, we present the individual element test (IET) of Bergan and Hanssen in an expanded context that encompasses several important classes of new elements. The relationship of the IET to the multielement forms A, B and C of the patch test and to the single element test are clarified.

\section{BACKGROUND}

The patch test for convergence is a fascinating area in the development of nonconforming finite element methods. It grew up of the brilliant intuition of Bruce Irons. Initially developed in the mid-1960s at Rolls Royce and then at the Swansea group headed by Olek Zienkiewicz, by the early 1970 s the test had became a powerful and practical tool for evaluating and checking nonconforming elements. And yet today it remains a controversial issue: accepted by most finite element developers while ignored by others, welcomed by element programmers, distrusted by mathematicians. For tracing down the origins of the test there is no better source than a 1973 survey article by Irons and Razzaque [12]. Added remarks to the quoted material are inserted in footnotes, and reference numbers have been altered to match those of the present paper.

Origins of the Patch Test

In 1965 even engineering intuition dared not predict the behavior of certain finite elements. Experience force those engineers who doubted it to admit that interelement continuity was important: the senior author ${ }^{1}$ believed that it was necessary for convergence. It is not known which ideas inspired a numerical experiment by Tocher and Kapur [25], which demonstrated convergence within $0.3 \%$ in a biharmonic problem of plate bending, using equal rectangular elements with $1, x, y, x^{2}, x y, y^{2}, y^{2}, x^{3}, x^{2} y, x y^{2}, y^{3}$, and $x^{3} y$ and $x y^{3}$, as functional basis. The nodal variable of this Ari Adini rectangle [1] are $w, \partial w / \partial x$ and $\partial w / \partial y$ at the four corners, and this element guarantees only $C^{0}$ conformity.

1 Bruce Irons 
Some months later, research at Rolls-Royce on the Zienkiewicz nonconforming triangle [2], - a similar plate-bending element ${ }^{2}$ - clarified the situation. Three elements with $C^{1}$ continuity were simultaneously available, and, because the shape function subroutine used for numerical integration had been exhaustively tested, the results were trustworthy. It was observed: (a) that every problem giving constant curvature over the whole domain was accurately solved by the conforming elements whatever the mesh pattern, as was expected, and (b) that the nonconforming element was also successful, but only for one particular mesh pattern. ${ }^{3}$

Thus the patch test was born. For if the external nodes of any sub-assembly of a successful assembly of elements are given prescribed values corresponding to an arbitrary state of constant curvature, then the internal nodes must obediently take their correct values. (An internal node is defined as one completely surrounded by elements.) Conversely, if two overlapping patches can reproduce any given state of constant curvature, they should combine into a larger successful patch, provided that every external node lost is internal to one of the original patches. For such nodes are in equilibrium at their correct values, and should behave correctly as internal nodes of the extended patch. In an unsuccessful patch test, the internal nodes take unsuitable values, which introduce interelement discontinuities. The errors in deflection may be slight, but the errors in curvature may be $\pm 20 \%$. We must recognize two distinct types of errors:

(i) The finite element equations would not be exactly satisfied by the correct values at the internal nodes - in structural terms, we have disequilibrium;

(ii) The answers are nonunique because the matrix of coefficients $\mathbf{K}$ is semidefinite.

Role of the Patch Test

Clearly the patch test provides a necessary condition for convergence with fine mesh. We are less confident that it provides a sufficient condition. The argument is that if the mesh is fine, the patches are also small. Over any patch the correct solution gives almost uniform conditions to which the patch is known to respond correctly - provided that the small perturbations from uniform conditions do not cause a disproportionate response in the patch: we hope to prevent this by insisting that $\mathbf{K}$ is positive definite.

The patch test is invaluable to the research worker. Already, it has made respectable

(i) Elements that do not conform,

(ii) Elements that contain singularities,

(iii) Elements that are approximately integrated,

(iv) Elements that have no clear physical basis.

In short, the patch test will help a research worker to exploit and justify his wildest ideas. It largely restores the freedom enjoyed by the early unsophisticated experimenters.

The late 1960s and early 1970 s were a period of unquestionable success for the test. That optimism is evident in the article quoted above, and prompted Gilbert Strang to develop a mathematical version popularized in the Strang-Fix textbook [21].

Confidence was shaken in the late 1970 s by several developments. Numerical experiments, for example, those of Sander and Beckers [20] suggested that the test is not necessary for convergence, thus disproving Irons' belief stated above. Then a counterexample by Stummel [22] purported to show that the test is not even sufficient. ${ }^{4}$ This motivated

2 This element is that identified by 'BCIZ' in the present paper

3 The bending element test referred to in this sentence appears in the Addendum to [2]. This Addendum was not part of the original paper presented at the First Wright-Patterson Conference held in September 1965; it was added to the Proceedings that appeared in 1966. The name "patch test" will not be found there; see the Appendix of [21] for further historical details.

4 Stummel has constructed [23] a generalized patch test that is mathematically impeccable in that it provides necessary and sufficient conditions for convergence. Unfortunately such test lacks important side benefits of Irons' patch test, such as element checkout by computer, because it is administered 
defensive responses by Irons [13] shortly before his untimely death, and by Taylor, Simo, Zienkiewicz and Chan [24]. These papers tried to set out the engineering version of the test on a more precise basis.

Despite these ruminations many questions persist, as noted in the lucid review article by Griffiths and Mitchell [11]. Some of them are listed below.

Q1. What is a patch? Is it the ensemble of all possible meshes? Are some meshes excluded? Can these meshes contain different types of elements?

Q2. The test was originally developed for harmonic and biharmonic compressible elasticity problems, for which the concept of "constant strains" or "constant curvatures" is unambiguous. But what is the equivalent concept for shells? Even Reissner-Mindlin plates (which lead to the so-called $C^{0}$ elements) pose difficulties.

Q3. What are the modifications required for incompressible media? Is the test applicable to dynamic or nonlinear problems?

Q4. Are single-element versions of the test equivalent to the conventional, multielement versions?

Q5. Is the test restricted to nonconforming assumed-displacement elements? Can it be extended to encompass assumed-stress or assumed-strain mixed and hybrid elements? (For initial attempts in this direction, see [10])

The following treatment is aimed primarily at answering the last two questions. No position as to the mathematical relevance of the test is taken.

\section{THE INDIVIDUAL ELEMENT TEST}

Because of practical difficulties incurred in testing all possible patches there have been efforts directed toward translating the original test into statements involving a single element. These will be collectively called one-element tests.

The first step along this path was taken by Strang [21], who using integration by parts recast the original test in terms of "jump" contour integrals over element interfaces. An updated account is given by Griffiths and Mitchell [11], who remark that Strang's test can be passed in three different ways:

JCS: Jump integrals cancel over common sides of adjacent elements (e.g. DeVeubeke's 3-midside-node triangle, Morley's plate elements).

JOS: Jump integrals cancel over opposite element sides (e.g. Wilson's incompatible plane rectangle [26]).

JEC: Jump integrals cancel over the element contour (see examples in [11]).

Another important development, not so well publicized as Strang's, was undertaken by Bergan and coworkers at Trondheim over the period 1975-1984. The so called individual

as a mathematically limiting process in function spaces. Furthermore, it does not apply to a mixture of different element types, or to situations such as a side shared by more than two elements. 
element test, or IET, was proposed by Bergan and Hanssen [4] in 1975. The underlying goal was to establish a test that could be directly carried out on the stiffness equations of a single element - an obvious improvement over the multielement form. In addition the test was to be constructive, i.e., used as an a priori guide during element formulation, rather than as a post-facto check.

The IET has a simple physical motivation: to demand pairwise cancellation of tractions among adjacent elements that are subjected to a common uniform stress state. This is precisely the 'JCS' case of the Strang test noted above. Because of this inclusion, the IET is said to be a strong version of the patch test in the following sense: any element passing the IET also verifies the conventional multielement form of the patch test, but the converse is not necessarily true.

The IET has formed the basis of the free formulation (FF) later developed by Bergan and Nygård [6]. It has also played an important part in the development of high performance finite elements undertaken by the authors $[7-9,15-18]$.

In an important paper written in response to Stummel's counterexample, Taylor, Simo, Zienkiewicz and Chan [24] defined multielement patch tests in more precise terms, introducing the so-called $\mathrm{A}, \mathrm{B}$ and $\mathrm{C}$ versions. They also discussed a one-element test called the "single element test," herein abbreviated to SET. They used the BCIZ plate bending element [2] to show that an element may pass the SET but fail multielement versions, and consequently that tests involving single elements are to be viewed with caution. In what follow we try to clarify this apparent contradiction and to establish precisely what the individual element test entails. In particular it is shown that the IET contains a crucial condition that the SET lacks, and that the two tests are not therefore equivalent.

Furthermore, we extend the IET to conditions beyond those considered by Bergan and Nygård by including elements with unknown internal displacement fields. The most important sources of such elements are: stress-assumed hybrids, and elements constructed through the assumed natural strain (ANS) and assumed natural deviatoric strain (ANDES) formulations.

\section{ASSUMPTIONS FOR ELEMENT CONSTRUCTION}

Suppose that we want to test an individual element of volume $V$ and boundary $S$ with exterior normal $\mathbf{n}$. The element satisfies the following assumptions.

A1. The element shares displacement degrees of freedom collected in $\mathbf{v}$ (the so called visible degrees of freedom) with adjacent elements. The boundary displacement field $\mathbf{d}$ is uniquely determined by $\mathbf{v}$ as

$$
\mathbf{d}=\mathbf{N}_{d} \mathbf{v}
$$

where $\mathbf{N}_{d}$ are boundary shape functions. 
The term "boundary displacement field" is meant to include normal derivatives (side rotations or slopes) in bending problems. More generally, in a problem governed by a variational principle of index $m>1, \mathrm{~d}$ includes normal derivatives up to the $(m-1)^{t h}$ order.

This assumption says nothing about the internal displacement field $\mathbf{u}$. In free-formulation elements $\mathbf{u}$ is known and agrees with $d$ only at the nodes. In the ANS $[3,14,19$,$] and ANDES [8,9,17,18]$ formulations, $\mathrm{u}$ is unknown because the deviatoric strain field $\epsilon_{h}$ introduced in A2 below is not generally integrable.

A2. The strain field $\epsilon$ within the element is expressible as

$$
\epsilon=\mathbf{B v}
$$

which admits the following decomposition into mean and deviatoric parts:

$$
\epsilon=\bar{\epsilon}+\epsilon_{h}=\overline{\mathbf{B}} \mathbf{v}+\mathrm{B}_{h} \mathbf{v}=\left(\overline{\mathrm{B}}+\mathrm{B}_{h}\right) \mathbf{v},
$$

where

$$
\bar{\epsilon}=\frac{1}{V} \int_{V} \epsilon d V, \quad \epsilon_{h}=\epsilon-\bar{\epsilon}
$$

We note that

$$
\int_{V} \mathbf{B}_{h} d V=\mathbf{0}, \quad \int_{V} \mathbf{A B}_{h} d V=\mathbf{0}
$$

in which $\mathbf{A}$ is an arbitrary matrix constant over the element.

Subscript $h$ stands for "high order." The strain field $\epsilon_{h}$ is not generally integrable, that is, associable with an internal displacement field $\mathbf{u}$ such that $\epsilon_{h}=\mathbf{D u}$, where $\mathbf{D}=\frac{1}{2}\left(\nabla+\nabla^{T}\right)$ is the symmetric vector gradient operator. On the other hand, the mean strain field $\bar{\epsilon}$, being constant, is integrable, as discussed under assumption A4.

A3. Suppose the element is under a constant stress state $\sigma_{0}$. Then a nodal force system $\mathbf{P}_{0}$ conjugate to $\mathbf{v}$ in the sense of virtual work develops. These forces are connected to $v$ through the relation

$$
\mathbf{p}_{0}=\mathrm{L} \sigma_{0}, \quad \mathrm{~L}=\int_{S} \mathbf{N}_{d n} d S,
$$

where $\mathbf{L}$ is called the force lumping matrix and $\mathbf{N}_{d n}$ denotes the projection of the shape functions $\mathbf{N}_{d}$ over the normal to the element side.

Matrix $\mathrm{L}$ was introduced by Bergan and coworkers in their studies leading to the free formulation $[5,6]$, and plays a crucial role in the individual element test.

A4. The constant stress field $\sigma_{0}$ is associated with a given displacement field called $\mathbf{u}_{r c}$, such that the associated strain and stress fields are

$$
\bar{\epsilon}^{u}=\mathrm{D}\left(\mathbf{u}_{r_{c}}\right), \quad \sigma^{0}=\mathbf{E} \bar{\epsilon}^{u},
$$

where $\mathbf{E}$ is the symmetric matrix of elastic moduli, assumed constant over the element. This constitutive assumption excludes incompressibility, which must receive special treatment. 
Field $\mathbf{u}_{r c}$ cannot be immediately linked to $\mathbf{v}$ because it spans a subspace of the possible boundary motions. We must start by expressing $\mathbf{u}_{r c}$ in the modal or generalized-coordinate form

$$
\mathbf{u}_{r c}=\mathbf{N}_{r c}^{q} \mathbf{q}_{r c} \text {, }
$$

where $\mathbf{N}_{r c}^{q}$ are modal functions and $\mathbf{q}_{r c}$ their amplitudes. The projection $\mathbf{v}_{r c}$ of $\mathbf{u}_{r c}$ over the space of boundary motions spanned by $\mathbf{v}$ can be most easily obtained by collocation, that is, evaluating $\mathbf{u}_{r c}$ at the nodal points. This process yields

$$
\mathbf{v}_{r c}=\mathbf{G}_{r c} \mathbf{q}_{r c}
$$

in which $\mathbf{G}_{r c}$ will generally be a rectangular matrix with more rows than columns.

Subscripts $r$ and $c$ mean that $\mathbf{u}_{r c}$ is supposed to include rigid-body and constant-strain modes. In mathematical terms, $\mathbf{u}_{r c}$ is a polynomial of degree $m-1$ when the variational index is $m$.

\section{THE STIFFNESS MATRIX}

Under the previous assumptions, the stiffness matrix is given by

$$
\mathbf{K}=\int_{V} \mathbf{B}^{T} \mathbf{E B} d V
$$

Using the strain decomposition (3), $\mathrm{K}$ splits as follows:

$$
\mathbf{K}=\mathbf{K}_{b}+\mathbf{K}_{h}=\int_{V} \overline{\mathbf{B}}^{T} \mathbf{E} \overline{\mathbf{B}} d V+\int_{V} \mathbf{B}_{h}^{T} \mathbf{E B}_{h} d V=V \overline{\mathbf{B}}^{T} \mathbf{E} \overline{\mathbf{B}}+\int_{V} \mathbf{B}_{h}^{T} \mathbf{E B}_{h} d V
$$

because of the energy orthogonality condition

$$
\int_{V} \overline{\mathbf{B}}^{T} \mathbf{E B}_{h} d V=\mathbf{0}
$$

which results on taking $\mathbf{A}=\overline{\mathbf{B}}^{T} \mathbf{E}$ on the second of (5). Matrices $\mathbf{K}_{b}$ and $\mathbf{K}_{h}$ receive the name of basic stiffness and higher order stiffness, respectively.

\section{FIRST CONDITION: CONSTANT STRAIN STATES}

Bergan and Nygård [6] state two constraints for $\mathrm{FF}$ elements, which taken together represent the satisfaction of the IET. The first one is

$$
\mathrm{K} \mathbf{v}_{r c}=\mathrm{L} \sigma_{0}
$$

which is essentially an equilibrium statement at the element level. Premultiplying (13) by $\mathbf{v}_{r c}^{T}$ we get $\mathbf{v}_{r c}^{T} \mathbf{K} \mathbf{v}_{r c}=\mathbf{v}_{r c}^{T} \mathbf{L} \sigma_{0}$, which on introducing (9) and (11) becomes

$$
\mathbf{q}_{r c}^{T} \mathbf{G}_{r c}^{T} \overline{\mathbf{B}}^{T} \mathbf{E} \overline{\mathbf{B}} \mathbf{G}_{r c} \mathbf{q}_{r c} V+\mathbf{q}_{r c}^{T} \mathbf{G}_{r c}^{T} \mathbf{K}_{h} \mathbf{G}_{r c} \mathbf{q}_{r c}=\mathbf{q}_{r c}^{T} \mathbf{G}_{r c}^{T} \mathbf{L} \boldsymbol{\sigma}_{0} .
$$


If we request that $\mathbf{K}_{h}$ cannot contribute to the internal energy under a constant strain state we must have $\mathbf{G}_{r c}^{T} \mathbf{K}_{h} \mathbf{G}_{r c}=\mathbf{0}$, or, since $\mathbf{K}_{h}=\int_{V} \mathbf{B}_{h} \mathbf{E B}_{h} d V$,

$$
\mathbf{B}_{h} \mathbf{G}_{r c}=\mathbf{0},
$$

This may be called the higher order strain cancellation condition. Taking into account that $\sigma_{0}=\mathrm{EB}_{r c}^{q} \mathrm{q}_{r c}$, where $\mathrm{B}_{r c}^{q}=\mathrm{D}\left(\mathrm{N}_{r c}^{q}\right)$, the above equation becomes $\overline{\mathrm{B}}^{T} \mathbf{E} \overline{\mathrm{B}} \mathrm{G}_{r c}=$ $V^{-1} \mathrm{LEB}_{r c}^{q}$. This can be split into $\overline{\mathbf{B}}^{T}=V^{-1} \mathbf{L}$ and $\overline{\mathbf{B}} \mathrm{G}_{r c}=\mathbf{B}_{r c}^{q}$. Replacing the former in the latter we obtain

$$
V^{-1} \mathbf{L}^{T} \mathbf{G}_{r c}=\mathbf{B}_{r c}^{q} .
$$

These conditions were introduced by Bergan and Nygård [6] in the context of the free formulation. They state that equation (16) should be used to check that the matrix $L$ is correct. Then (16) is the first consistency constraint on L. Equations (15) and (16) are necessary in order that a single element, which is in equilibrium, be capable of copying a constant strain state. To prove that they are also sufficient conditions is straightforward.

An important consequence of (16) can be investigated as follows. Rewrite it as $V^{-1} \mathbf{L}^{T} \mathbf{G}_{r c} \mathbf{q}_{r c}=\mathrm{Du}_{r c}$. Multiplying both sides by $\boldsymbol{\sigma}_{0}^{T}$ and integrating over the element volume we obtain

$$
\sigma_{0}^{T} \mathrm{~L}^{T} \mathrm{G}_{r c} \mathbf{q}_{r c}=\int_{V} \sigma_{0}^{T} \mathrm{Du}_{r c} d V .
$$

Integration by parts of the right hand side yields

$$
\sigma_{0}^{T} \mathbf{L}^{T} \mathbf{G}_{r c} \mathbf{q}_{r c}=\sigma_{0}^{T} \int_{S} \mathbf{N}_{u n} d S \mathbf{q}_{r c}
$$

where $\mathbf{N}_{u n}$ are the the projections of the modal functions over the normal to the element side. From the definition of $\mathrm{L}$ in (6) we conclude that

$$
\int_{S} \mathbf{N}_{d n}^{T} d S \mathbf{G}_{r c} \mathbf{q}_{r c}=\int_{S} \mathbf{N}_{u n} d S \mathbf{q}_{r c}
$$

This result may be stated as follows: the force lumping produced by the boundary displacement field should be energy consistent (in the sense of virtual work) with that produced by the displacement field $\mathbf{u}_{r c}$ over the element side. Although $\mathbf{B}_{r c}^{q}$ is unique for a given problem, since $\mathbf{G}_{r c}$ is generally a rectangular matrix, equation (16) clearly shows that $\mathbf{L}$ is not necessarily unique. Examples that illustrate this property may be found in [17]. L is unique for simplex elements where we have the same number of nodal connectors $\mathbf{v}$ and $r c$-modal amplitudes $\mathbf{q}_{r c}$, because in this case $\mathbf{G}_{r c}$ is square and non-singular. For these elements the total and basic stiffness matrices coalesce. An obvious example is provided by the constant strain triangle (CST). 


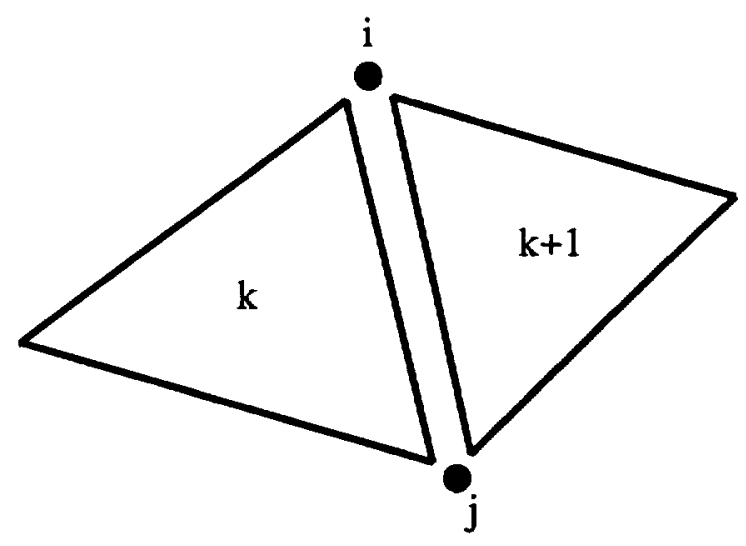

Figure 1: A common side $i-j$ shared by two elements

\section{SECOND CONDITION: PAIRWISE FORCE CANCELLATION}

Quoting from [4]: "The basis for the individual element test is that the element, when interacting with its neighbors, should be capable of identically reproducing an arbitrary rigid-body/constant strain field ... The interelement forces transferred at nodes should cancel out in a pairwise manner for adjacent elements during such state." This establishes a second key constraint on $\mathbf{L}$.

Suppose we have a side $i-j$ joining elements $k$ and $k+1$, as illustrated in Figure 1. The second condition requires that

$$
\int_{i}^{j} \mathrm{~N}_{d n}^{k} d S=\int_{i}^{j} \mathrm{~N}_{d n}^{k+1} d S .
$$

The easiest way of enforcing this condition is by choosing a boundary displacement $\mathbf{d}$ that is uniquely defined over $i-j$ by degrees of freedom on that interface.

This rule can be extended to cases in which more than two elements share a side, as is the case in many practical structures. Note that (20) does not involve the internal displacement field in any way. Consequently it establishes the mixability of elements of different types (for example, FF with ANDES elements). The SET discussed in [24] omits this important condition.

\section{MULTIELEMENT PATCH TESTS AND THE IET}

Bergan and coworkers called conditions (13) and (20) the IET. We now prove that if the element under consideration satisfies these conditions, it will also pass the so-called forms $\mathrm{A}$ and $\mathrm{B}$ of the multielement patch test [24]. Furthermore, if the element is rank sufficient it will also pass form $\mathrm{C}$.

Let us consider the assemblage of elements shown in Figure 2 as a patch. The global displacement field consistent with a constant strain field is $\mathbf{v}_{r c}^{g}=G_{r c}^{g} \mathbf{q}_{r c}$ The stiffness matrix of the $k^{\text {th }}$ element satisfies equation (13), or its equivalent global form

$$
\left(\mathbf{P}^{k}\right)^{T} \mathbf{K}^{k} \mathbf{P}^{k} \mathbf{v}_{r c}^{g}=\left(\mathbf{P}^{k}\right)^{T} \mathbf{L}^{k} \sigma_{0}
$$




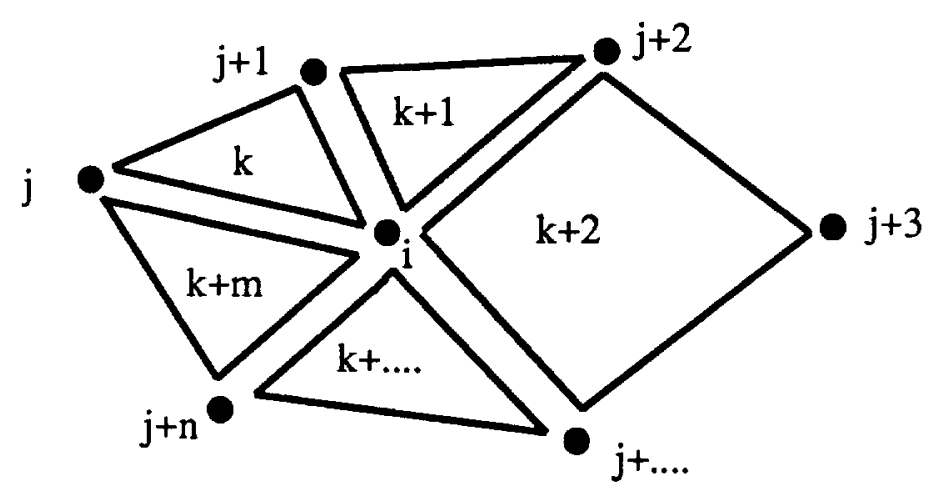

Figure 2: An assemblage of elements

where $\mathbf{P}^{k}$ are Boolean localization matrices. Upon assembly we obtain

$$
\mathbf{K}^{g} \mathbf{v}_{r c}^{g}=\mathbf{L}^{g} \sigma_{0}=\mathbf{p},
$$

but because of the satisfaction of equation (20) the force vector $\mathbf{p}$ has only components in nodes $j, \ldots j+n$. Then, for the $i^{t h}$ internal degree of freedom we have

$$
K_{i j}^{g} v_{r c j}^{g}=p_{i}=0,
$$

which is the statement of the form $A$ of the patch test. If an element satisfies $A$, form $B$ is also satisfied because from (22) we can obtain the displacement of the internal node $i$ as:

$$
v_{r c_{i}}=\left(K_{i i}^{g}\right)^{-1}\left(p_{i}-K_{i j} v_{r c_{j}}^{g}\right), \quad j \neq i
$$

Because the element satisfies (13), $\mathbf{v}_{r c}$ can be obtained if upon removing the rigid body motions $\mathrm{K}^{k}$ is nonsingular and can be inverted. Consequently $\mathrm{K}^{k}$ should be rank sufficient in order to satisfy form C.

\section{CONCLUSIONS}

It has been shown that the IET constraints plus rank sufficiency provide sufficient conditions to pass any form of the multielement patch test. The main practical advantages of the IET are:

1. By applying rules (13) and (20) elements can be constructed that will pass any multielement patch test a priori, provided that they are rank sufficient, while being capable of copying constant strain states. No such possibility exists in the conventional patch test, which must be necessarily applied a posteriori.

2. Element mixability is immediately established. 
3. A "surgical operation" can be established to "sanitize" elements that fail the IET, as discussed in the Appendix of [17]. The operation essentially amounts to the replacement of the basic stiffness.

The price paid for these advantages is that the test is occasionally stronger than strictly necessary. For example, the BCIZ nonconforming triangle [2] fails the IET but passes the multielement test for certain mesh configurations.

A potential difficulty in the application of the IET to existing elements is the need for extracting the force-lumping matrix $\mathbf{L}$. This matrix may not be readily available and, as mentioned in Section 5 , is not necessarily unique.

Finally, as remarked in several places, the present statement of the IET is not restricted to the free formulation, and has actually been used in this expanded form for constructing high-performance elements based on the ANDES formulation $[8,9,17,18]$.

\section{ACKNOWLEDGEMENT}

The work reported here has been supported by NASA Langley Research Center under Grant NAG1-756.

\section{REFERENCES}

1. ADINI, A. - Analysis of Shell Structures by the Finite Element Method, Ph. D. Dissertation, Dept. of Civil Engineering, University of California, Berkeley, CA, 1961.

2. BAZELEY, G.P., CHEUNG, Y.K., IRONS, B.M. and ZIENKIEWICZ, O.C. - Triangular Elements in Plate Bending - Conforming and Nonconforming Solutions, Proceedings 1st Conference on Malrix Methods in Structural Mechanics, AFFDL-TR-66-80, Air Force Institute of Technology, Dayton, Ohio, 1966, p. 547.

3. BATHE, K.J. and DVORKIN, E.N. - A Four-node Plate Bending Element Based on Mindlin/Reissner Plate Theory and a Mixed Interpolation, Int. J. Numer. Meth. Engrg., 21, 367-383 (1985)

4. BERGAN, P.G. and HANSSEN, L. - A New Approach for Deriving 'Good' Finite Elements, MAFELAP II Conference, Brunel University, 1975, in The Mathematics of Finite Elements and Applications - Volume II, ed. by J. R. Whiteman, Academic Press, London, 1976.

5. BERGAN, P.G. - Finite Elements Based on Energy Orthogonal Functions, Int. J. Num. Meth. Engrg., 15, 1141-1555 (1980)

6. BERGAN, P.G. and NYGÅD, M.K. - Finite Elements with Increased Freedom in Choosing Shape Functions, Int. J. Num. Meth. Engrg., 20, 643-664 (1984)

7. FELIPPA, C.A. - Parametrized Multifield Variational Principles in Elasticity: II. Hybrid Functionals and the Free Formulation, Communications in Applied Numerical Methods, $\underline{5}$, 79-88 (1989)

8. FElipPA, C.A. and Militello, C. - Variational Formulation of High Performance Finite Elements: Parametrized Variational Principles, Computers \& Structures, $\underline{\text { 36, }}$ 1-11 (1990)

9. FELIPPA, C.A. and MILITELLO, C. - 'Developments in Variational Methods for High Performance Plate and Shell Elements', in Analytical and Computational Models for Shells, CED Vol. 3, Eds. A. K. Noor, T. Belytschko and J. C. Simo, The American Society of Mechanical Engineers, ASME, New York, 1989, p. 191.

10. FRAEIJS DE VEUBEKE, B.M. - Variational Principles and the Patch Test, Int. J. Numer. Meth. Engrg, $\underline{\mathbf{8}}, 783-801$ (1974) 
11. GRIFFITHS, D.F . and MITCHELL, A.R. - 'Nonconforming Elements', in The Mathematical Basis of Finite Element Methods, Ed. Griffiths, D. F., Clarendon Press, Oxford, 1984, p. 41.

12. IRONS, B.M. and RAZZAQUE, A. - 'Experiences with the Patch Test for Convergence of Finite Elements', in The Mathematical Foundations of the Finite Element Method with Applications to Partial Differential Equations, Ed. Aziz, A. K., Academic Press, New York, 1972, p. 557.

13. IRONS, B.M. and LOIKKANEN, M. - An Engineer's Defense of the Patch Test, Int. J. Numer. Meth. Engrg., 19, 1391-1401 (1983)

14. MacNEAL, R.H. - Derivation of Element Stiffness Matrices by Assumed Strain Distribution, Nuclear Engrg. Design, 70, 3-12 (1978)

15. MiLitello, C. and FELIPPA, C.A. - A Variational Justification of the Assumed Natural Strain Formulation of Finite Elements: I. Variational Principles, Computers \& Structures, $\underline{\mathbf{3 4}}, \mathbf{4 3 1 - 4 3 8}$ (1990)

16. MILITELLO, C. and FELIPPA, C.A. - A Variational Justification of the Assumed Natural Strain Formulation of Finite Elements: II. The Four Node $C^{0}$ Plate Element, Computers \& Structures, $\underline{\mathbf{3 4}}$, 439-444 (1990)

17. Militello, C. and FELIPPA, C.A. - The First ANDES Elements: 9-DOF Plate Bending Triangles, to appear in Comp. Meth. Appl. Mech. Engrg. (1991)

18. Militello, C. - Application of Parametrized Variational Principles to the Finite Element Method, Ph. D. Dissertation, Department of Aerospace Engineering Sciences, University of Colorado, Boulder, CO, 1991.

19. PARK, K.C. and STANLEY, G.M. - A Curved $C^{0}$ Shell Element Based on Assumed NaturalCoordinate Strains, Journal of Applied Mechanics, $\underline{\mathbf{5 3}}, 278-290$ (1986)

20. SANDER, G. and BECKERS, P. - 'The Influence of the Choice of Connectors in the Finite Element Method', in The Mathematical Aspects of the Finite Element Method, Lecture Notes in Mathematics, Vol. 606, Springer-Verlag, Berlin, 1977, p. 316.

21. STRANG, G. and FIX, G. - An Analysis of the Finite Element Method, Prentice-Hall, Englewood Cliffs, N.J., 1973.

22. STUMMEL, F. - The Limitations of the Patch Test, Int. J. Numer. Meth. Engrg., 15, 177-188 (1980)

23. STUMMEL, F. - The Generalized Patch Test, SIAM J. Numer. Anal., 16, 449-471 (1979)

24. TAYLOR, R.L., SIMO, J.C., ZIENKIEWICZ, O.C. and CHAN, A.C. - The Patch Test: A Condition for Assessing FEM Convergence, Int. J. Numer. Meth. Engrg, 22, 39-62 (1986)

25. TOCHER, J.L. and KAPUR, K.K. - Discussion of 'Basis for derivation of matrices for the direct stiffness method' by R. J. Melosh, J. AIAA, $\underline{3}, 1215-1216$ (1965)

26. WILSON, E.L., TAYLOR, R.L., DOHERTY, W.P. and GHABOUSSI, J. - 'Incompatible Displacement Models', in Numerical and Computer Models in Structural Mechanics, Eds. S. J. Fenves et.al., Academic Press, New York, 1973, p. 43. 